\title{
Cosmic rays in early star-forming galaxies and their effects on the interstellar medium
}

\author{
Ellis R. Owen ${ }^{* a, b}$ Kinwah Wu, ${ }^{a, d}$ Pooja Surajbali ${ }^{c}$ and Idunn B. Jacobsen ${ }^{a}$ \\ ${ }^{a}$ Mullard Space Science Laboratory, University College London, Holmbury St. Mary, Dorking, \\ Surrey, RH5 6NT, United Kingdom \\ ${ }^{b}$ Department of Physics, National Tsing Hua University, Hsinchu, Taiwan (ROC) \\ ${ }^{c}$ Max-Planck-Institut für Kernphysik, Saupfercheckweg 1, Heidelberg 69117, Germany \\ ${ }^{d}$ School of Physics, University of Sydney, NSW 2006, Australia \\ E-mails: ellis.owen.12@ucl.ac.uk, kinwah.wu@ucl.ac.uk, \\ pooja.surajbaliempi-hd.mpg.de, idunn.jacobsen.09@ucl.ac.uk
}

\begin{abstract}
Galaxies at high redshifts with strong star formation are sources of high-energy cosmic rays. These cosmic rays interact with the baryon and radiation fields of the galactic environment via photo-pair, photo-pion and proton-proton processes to produce charged and neutral pions, neutrons and protons. The cosmic rays thereby deposit energy into the interstellar medium (ISM) as they propagate. We show how energy transport and deposition by ultra high-energy cosmic rays is regulated by the evolution of the galaxy, in particular by the development of the galactic magnetic field. We show how the particle-driven energy deposition can influence the thermal evolution of the host and its surroundings. Using a parametric protogalaxy model, we calculate the heating effect on the ISM as the cosmic rays are increasingly confined by the magnetic evolution of the galaxy.
\end{abstract}

35th International Cosmic Ray Conference - ICRC2017

10-20 July, 2017

Bexco, Busan, Korea

* Speaker. 


\section{Introduction}

High-redshift star forming protogalaxies are thought to be abundant in ultra-high-energy (UHE) cosmic ray (CR) particles. The star formation rates (SFR) of such galaxies yield high rates of supernova (SN) explosions that provide ample extreme environments in compact objects (e.g. fast spinning neutron stars and accreting black holes) and supernova remnants (SNR). These are considered likely regions in which $\mathrm{CR}$ acceleration may reach energies above $10^{17} \mathrm{eV}$ (see e.g. $[1,2,3,4]$ ).

In this work we employ a generic spherical protogalaxy model of $10^{6}$ stars, intended as a parametric mean. It is defined by a density field (of characteristic radius $1 \mathrm{kpc}$ and peak density 10 $\mathrm{cm}^{-3}$ following a Dehnen profile [5]), a magnetic field (described by the model introduced in [6], but spatially following the density profile) and a radiation field which is dominated by the cosmic microwave background (CMB). We set our model to exhibit extreme starburst characteristics with a high SFR of $1000 \mathrm{M}_{\odot} \mathrm{yr}^{-1}$. From this, a SN rate can be estimated. A typical SN explosion is set to have an energy of $10^{51} \mathrm{erg}$, of which a fraction of $\xi=0.1$ is transferred into CR proton energy. We model primary $\mathrm{CR}$ production as a population of high-energy protons following a power-law distribution in energy above a $\mathrm{GeV}$, extending up to $10^{11} \mathrm{GeV}$ with spectral index -2.1 , similar to CR source regions in the Milky Way [1, 7]. The CR luminosity is determined by the SFR (via the $\mathrm{SN}$ rate, assuming stars of mass $>8 \mathrm{M}_{\odot}$ yield $\mathrm{SN}$ explosions, being around $4 \%$ of the stellar population if adopting a Salpeter IMF of index 2.35).

\section{Cosmic Ray Interactions}

More than $99 \%$ of the energy of a typical CR population is contained in the high-energy component, above $\mathrm{a} \mathrm{GeV}^{1}$ (see, e.g. [8]), so these are most likely to drive a heating effect. While high-energy CRs can deposit their energy by collisional energy transfer/ionization, this is relatively inefficient. A $0.5 \mathrm{GeV}$ CR proton would only lose around $2.5 \%$ of its energy by this mechanism over a Hubble time at $z=7$ [9]. Above a threshold of around a $\mathrm{GeV}$, pion-producing hadronic interactions with the radiation and baryon fields of the galaxy are a much more effective means by which CRs can deposit energy into their surroundings.

The interaction of CR protons with the radiation field - $\mathrm{p} \gamma$ interactions - can proceed via two fundamental channels. These are: (1) a Bethe-Heitler pair production process [10] proceeding as $\mathrm{A} \gamma \rightarrow \mathrm{A}^{\prime} l^{+} l^{-}$, where $\mathrm{A}$ and $\mathrm{A}^{\prime}$ are nucleons (i.e. the incident $\mathrm{CR}$ before and after the interaction), and $l^{+}$and $l^{-}$are charged leptons (at the energies of interest, around $90 \%$ of the produced secondaries are electrons and positrons, with around $10 \%$ being heavier counterparts); and (2) a photo-pion process proceeding as a resonant single pion production through the formation of $\Delta^{+}$ particles $\left(\mathrm{p} \gamma \rightarrow \Delta^{+}\right)$. These $\Delta^{+}$decay through two major channels, where charged and neutral pions are produced. These pions decay as $\mathrm{p} \pi^{0} \rightarrow \mathrm{p} 2 \gamma$ and $\mathrm{n} \pi^{+} \rightarrow \mathrm{n} \mu^{+} v_{\mu}$ where $\mu^{+} \rightarrow \mathrm{e}^{+} v_{\mathrm{e}} \bar{v}_{\mu}$ (see [11]). The branching ratios for the $\left(\Delta^{+} \rightarrow \pi^{0}\right)$ and $\left(\Delta^{+} \rightarrow \pi^{+}\right)$channels are $2 / 3$ and $1 / 3$ respectively. The direct production of pions is less dominant, occurring at a fraction of the rate of the above interaction, so is ignored in this analysis. CRs above $0.28 \mathrm{GeV}$ also undergo losses by interactions with the baryon field of the galaxy. These pion-production losses proceed as $\mathrm{pp} \rightarrow \mathrm{p} \Delta^{+}$ and $\mathrm{pp} \rightarrow \mathrm{n} \Delta^{++}$with $\Delta^{+}$and $\Delta^{++}$baryons as the resonances $[12,13]$.

\footnotetext{
${ }^{1}$ Note that these energies refer to the CR kinetic energy, not total energy.
} 
In these channels, the charged pion decay is largely responsible for the deposition of energy in the medium. This is because they mostly decay to produce leptons (which scatter strongly and cool quickly in an ISM environment) and neutrinos via a weak interaction $\left(\pi^{+} \rightarrow \mu^{+} v_{\mu} \rightarrow \mathrm{e}^{+} v_{\mathrm{e}} \bar{v}_{\mu} v_{\mu}\right.$ and $\pi^{-} \rightarrow \mu^{-} \bar{v}_{\mu} \rightarrow \mathrm{e}^{-} \bar{v}_{\mathrm{e}} v_{\mu} \bar{v}_{\mu}$ ) on a timescale of $2.6 \times 10^{-8} \mathrm{~s}$. The $\pi^{0}$ production leads to $\gamma$-ray emission in an electromagnetic process $\left(\pi^{0} \rightarrow 2 \gamma\right)$ over shorter timescales of $8.5 \times 10^{-16} \mathrm{~s}$.

\subsection{Interaction Cross Sections and Path Lengths}

The energy loss driven by an interaction can be characterised in terms of its inelastic cross section which is specific to each interaction. We may express an interaction cross section as a function of the invariant normalised CR energy, $\varepsilon_{\mathrm{r}}=\gamma_{\mathrm{p}}\left(1-\beta_{\mathrm{p}}\right) \varepsilon$. Here $\mu=\cos \theta$ for $\theta$ as the angle between the momentum vectors of the $\mathrm{CR}$ and target photon or proton, $\varepsilon$ is the energy of the target photon or proton normalised to electron rest mass (being $h v / m_{\mathrm{e}} c^{2}$ and $m_{\mathrm{p}} / m_{\mathrm{e}}$ respectively) and $\gamma_{p}$ is the Lorentz factor of an incident CR proton, i.e. $E_{\mathrm{CR}}=\gamma_{\mathrm{p}} m_{\mathrm{p}} c^{2}$. The cross section for photo-pair production may be approximated as

$$
\hat{\sigma}_{\gamma \mathrm{e}}\left(\varepsilon_{\mathrm{r}}\right) \approx\left\{\frac{7}{6 \pi} \alpha_{\mathrm{f}} \ln \left[\frac{\varepsilon_{\mathrm{r}}}{k_{\gamma \mathrm{e}}}\right]\right\} \sigma_{\mathrm{T}}
$$

with $k_{\gamma \mathrm{e}}$ taking a value of $\approx 6.7^{2}$. Here $\alpha_{\mathrm{f}}$ is the fine structure constant and $\sigma_{\mathrm{T}}$ is the Thomson scattering cross section. Without losing generality we fix $k_{\gamma \mathrm{e}}=6.7$ in our calculations. In line with $[18,19]$, the photo-pion cross section can be estimated as $\hat{\sigma}_{\gamma \pi} \approx \hat{\sigma}_{\gamma \pi}^{*} \mathscr{H}\left(\varepsilon_{\mathrm{r}}-\varepsilon_{\mathrm{th}}\right)$, where $\mathscr{H}(\ldots)$ is the Heaviside step function. In our calculations we adopt $\hat{\sigma}_{\gamma \pi}^{*}=70 \mu \mathrm{b}$ and $\varepsilon_{\text {th }}=390$. We adopt the analytic cross section parameterisation prescribed by [20] for the pp interaction.

We can account for the relative importance of these various processes as a function of energy by characterising the CR losses in terms of the effective mean free path over which a CR proton loses its energy. Following [21, 18] for the photo-pair interaction, this path length is

$$
r_{\gamma \mathrm{e}}\left(\gamma_{\mathrm{p}}\right) \approx \mathscr{R}_{\mathrm{m}} \frac{1125}{14} \frac{\lambda_{\mathrm{C}}^{3} \gamma_{\mathrm{p}}^{3} b^{5 / 3}}{\alpha_{\mathrm{f}} \sigma_{\mathrm{T}} \mathscr{F}_{\gamma \mathrm{e}}}
$$

in Mpc, where $\lambda_{\mathrm{C}}$ is the Compton wavelength, $\mathscr{R}_{\mathrm{m}}=\frac{m_{\mathrm{p}}}{m_{\mathrm{e}}}$ is the ratio of proton to electron mass, $b=m_{\mathrm{e}} \mathrm{c}^{2} / \gamma_{p} \mathrm{k}_{\mathrm{B}} T$ and

$$
\mathscr{F}_{\gamma \mathrm{e}}=\mathscr{C}(b)-\mathscr{D}(b) \ln \left[\frac{b k_{\gamma_{\mathrm{e}}}}{1.03}\right]-\left(\frac{b k_{\gamma_{\mathrm{e}}}}{2.62}\right)^{3 / 2} \ln \left[1-e^{-b}\right] .
$$

The functions $\mathscr{C}(b), \mathscr{D}(b)$ and $\mathscr{E}(b)$ result from standard integrals, with $\mathscr{C}(b)=0.74$ and $\mathscr{D}(b)=$ $\Gamma(5 / 2) \zeta(5 / 2)$ when $b \ll 1, \mathscr{C}(b)=b^{3 / 2} \ln (b) e^{-b}$ and $\mathscr{D}(b)=b^{3 / 2} e^{-b}$ when $b \gg 1$ [18]. The photo-pion interaction yields a path length of

$$
r_{\gamma \pi} \approx \frac{\lambda_{\mathrm{C}}^{3}}{16 \pi \hat{\sigma}_{\gamma \pi}^{*}}\left(\frac{m_{\mathrm{e}} \mathrm{c}^{2}}{k_{\mathrm{B}} T}\right)^{3} \mathrm{e}^{k \eta}\left[\sum_{k=1}^{\infty}\left(\frac{\eta}{k^{2}}+\frac{1}{k^{3}}\right)\right]^{-1},
$$

where $\eta=\varepsilon_{\mathrm{th}} m_{\mathrm{e}} \mathrm{c}^{2} / 2 \gamma_{\mathrm{p}} \mathrm{k}_{\mathrm{B}} T$.

\footnotetext{
${ }^{2}$ Note that this approximation follows from the treatment outlined in [14] - see also [15, 16, 17]
} 
The pp interaction path length can be reduced to the classical definition of the mean free path of a scattered particle, $r_{\mathrm{p} \pi}=\left(\hat{\mathrm{\sigma}}_{\mathrm{p} \pi} n_{\mathrm{p}}\right)^{-1}$, which is valid when the target baryon field is assumed to be at rest. This is reasonable given that the energy of the particles which make up the target baryon field are generally of much lower energy than the UHECRs.

We find that $\mathrm{p} \gamma$ interactions with the CMB dominate the CR losses due to the radiation fields. Overall, however, these are only important above energies of around $10^{19} \mathrm{eV}$. Below this, pp interactions govern the majority of the energy deposition and subsequent heating process by the UHECRs.

\subsection{Cosmic Ray Propagation}

High-energy CRs will travel at approximately the speed of light $c$ in free space, but in the presence of a sufficiently strong magnetic field $(B \sim \mu \mathrm{G})$, the propagation of CRs is practically a diffusive process, described by the transport equation:

$$
\frac{\partial n}{\partial t}=\nabla \cdot[D(E, r, t) \nabla n]+Q(r, E)
$$

where $n(E, r, t)$ is the local CR number density and $D(E, r, t)$ is the diffusion coefficient (units $\left.\mathrm{cm}^{2} \mathrm{~s}^{-1}\right) . Q(r, E)$ is a composite source term, consisting of an injec-

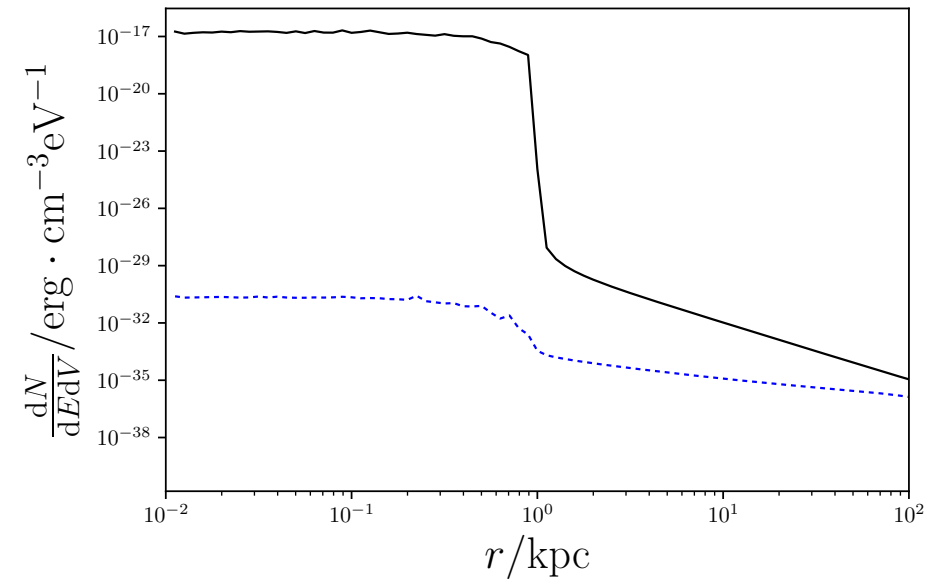

Figure 1: Diffusion profile after magnetic saturation (black). For comparison, we show the initial free-streaming case (blue). tion term and a loss term, which are functions of CR energy, $E$. If the CR loss is insignificant, the problem is essentially the transportation of a packet of CRs from the injection site across the system, i.e. the protogalaxy. Without losing generality, we may consider the system at first as an initial value problem (IVP) with $n(r, t=0)=Q=F_{\mathrm{CR}}(E) \cdot \mathscr{N}\left(r_{s}\right) \mathrm{erg} \mathrm{s}^{-1} \mathrm{eV}^{-1}$, where $r_{s}$ is the injection source location. The solution of this IVP is then integrated over time to yield the solution to the transport equation as

$$
N(E, r, t)=\frac{F_{\mathrm{CR}} \cdot \mathscr{N}\left(r_{s}\right) t}{(4 \pi D(E, r, t) t)^{3 / 2}}\left\{\frac{\left(r-r_{s}\right)^{2}}{4 D(E, r, t) t}\right\}^{-1 / 2} \Gamma\left(\frac{1}{2}, \frac{\left(r-r_{s}\right)^{2}}{4 D(E, r, t) t}\right)+\mathscr{C}(E, r)
$$

(in units of $\left.\mathrm{erg} \mathrm{cm}^{-3} \mathrm{eV}^{-1}\right)$, where $\mathscr{C}(E, r)$ is the constant of integration, which can be derived from the initial freely streaming $C R$ distribution. This solution corresponds to a continuous injection by a single source (of a size $\sim 0.01 \mathrm{pc}$ if it is a SNR, see, e.g. [22]). A Monte-Carlo (MC) method may be used to sum over all the source contributions within the host galaxy distribution. In this work we weight the CR source contribution by the density profile of the galaxy. This is because we assume that the $\mathrm{CR}$ sources are remnants of stars, which are ultimately derived from the gas profile from which stars form. Adopting a SFR of $1000 \mathrm{M}_{\odot} \mathrm{yr}^{-1}$ and a magnetic field saturating at $10 \mu \mathrm{G}$, we obtain a CR profile as shown in Fig. 1. 


\subsection{Cooling Losses}

CR protons and their secondary electrons can lose energy in the ISM of high redshift galaxies through synchrotron, inverse Compton and free-free emission mechanisms. We can quantify the relative importance of a cooling mechanism by considering the respective CR energy loss timescale. At high energies and high redshifts, inverse Compton losses dominate from scattering off CMB photons $[23,24]$. The typical loss timescale as a function of secondary CR electron energy by this process is given by

$$
\tau(E)=\frac{3 m_{\mathrm{e}}^{2} c^{3}}{4 \sigma_{\mathrm{T}} U_{\mathrm{CMB}} E}
$$

where $c$ is the speed of light, $m_{\mathrm{e}}$ is the mass of an electron and $U_{\mathrm{CMB}}$ is the energy density of the CMB,

$$
U_{\mathrm{CMB}}=\frac{8 \pi^{5} k_{\mathrm{B}}^{4}}{15 c^{3} h^{3}} T_{0}^{4}(1+z)^{4} .
$$

Here $h$ is the Planck constant, $T_{0}=2.73 \mathrm{~K}$ is the current CMB temperature [25], and $z$ is the redshift of interest - in this study, we take $z=7$.

\subsection{Sunyaev-Zel'dovich Effect}

Given the dominance of inverse Compton cooling of the CRs at high redshift, we may consider the impact of the resulting emission from these losses. The scattering of high-energy electrons and protons off CMB photons is known as the Sunyaev-Zel'dovich effect (SZE) [26]. In general, the inverse-Compton power per unit volume per energy is given by

$$
\frac{\mathrm{d} P(\varepsilon)}{\mathrm{d} \varepsilon}=\frac{\mathrm{d} E}{\mathrm{~d} V \mathrm{~d} t \mathrm{~d} \varepsilon}=\frac{N_{\mathrm{e}} 8 \pi^{2} r_{0}^{2}}{h^{3} c^{2}}\left[k_{\mathrm{B}} T_{0}(1+z)\right]^{(p+5) / 2} A(p) \Gamma\left(\frac{p+5}{2}\right) \zeta\left(\frac{p+5}{2}\right) \varepsilon^{-(p-1) / 2},
$$

in erg $\mathrm{cm}^{-3} \mathrm{~s}^{-1} \mathrm{eV}^{-1}[27,28]$, where $r_{0}$ is the classical electron radius, $p$ is the negative power law index of the CR spectrum and

$$
A(p)=2^{p+3} \frac{p^{2}+4 p+11}{(p+3)^{2}(p+5)(p+1)} .
$$

From equation 2.7, it can be seen that the SZ emission due to electrons dominates that by protons by around three orders of magnitude (due to their mass ratio). While we have assumed that primary CR electrons deposit their energy relatively close to their source (and so have ignored their contribution in the present work), we can account for the SZ emission due to the secondary CR electrons produced from the interaction showers of the primary CR protons.

We employ a secondary CR electron injection model based on the injection of primary CR protons $Q_{\mathrm{p}}\left(\gamma_{\mathrm{p}}\right)$, as in [23,29]. This gives a CR electron injection rate of

$$
Q_{\mathrm{e}}\left(\gamma_{\mathrm{e}}\right) \simeq \frac{\Upsilon}{6} \frac{400 m_{\mathrm{e}}}{m_{\mathrm{p}}} Q_{\mathrm{p}}\left(\gamma_{\mathrm{p}}\right)
$$

which is dependent on the energy of the primary CR by $\gamma_{\mathrm{p}}=E / m_{\mathrm{p}} c^{2}$ and where $\gamma_{\mathrm{e}}=E / m_{\mathrm{e}} c^{2}$. $\Upsilon$ is the fraction of energy of the original $\mathrm{CR}$ which is transferred to the electron and positron producing branches of the particle shower, taken to be 0.5 [30]. $N_{\mathrm{e}}$ can be estimated from $Q_{\mathrm{e}} * \tau_{\text {cool }} * \mathscr{X}(r)$. 
We can estimate the X-ray luminosity between $E_{\min }=100 \mathrm{eV}$ and $E_{\max }=100 \mathrm{keV}$ by considering the inverse-Compton energy that goes into this band. We take the emission is this band as the source of X-ray heating. The power emitted in this band per unit volume is

$$
P(\varepsilon)=\frac{N_{\mathrm{e}} 8 \pi^{2} r_{0}^{2}}{h^{3} c^{2}}\left[k_{\mathrm{B}} T_{0}(1+z)\right]^{(p+5) / 2} B(p) \Gamma\left(\frac{p+5}{2}\right) \zeta\left(\frac{p+5}{2}\right)\left[E_{\max }^{(3-p) / 2}-E_{\min }^{(3-p) / 2}\right],
$$

where $B(p)=2 A(p) /(3-p)$ and with the constraint that $p \neq 3$. For comparison, this indicates a total SZE X-ray luminosity of the star forming galaxy of up to around $10^{48} \mathrm{erg} \mathrm{s}^{-1}$ in the 0.5 to 8 $\mathrm{keV}$ band often used in observational studies. Local and distant starbursts exhibit X-ray luminosities of around 5 orders of magnitude lower than this (see [31]), perhaps indicative of the role played by heating-driven outflows and other CR escape mechanisms to reduce the true CR containment level. This more detailed modelling is left to future work.

\section{Heating Processes}

The heating at a position $r$ in a medium irradiated by $\mathrm{X}$-rays or CRs is calculated as the tendency of the medium to absorb radiation at that point (given by the absorption coefficient $\alpha(r)=n(r) \sigma)$ accounting for the attenuation along the particle or radiation paths. For the source distribution in the protogalaxy model considered here, we invoke an MC method in which the total source luminosity is split across an ensemble of points. Additionally, we introduce a scaling parameter, $\mathscr{X}(r)$, to account for the contained profile of CRs due to

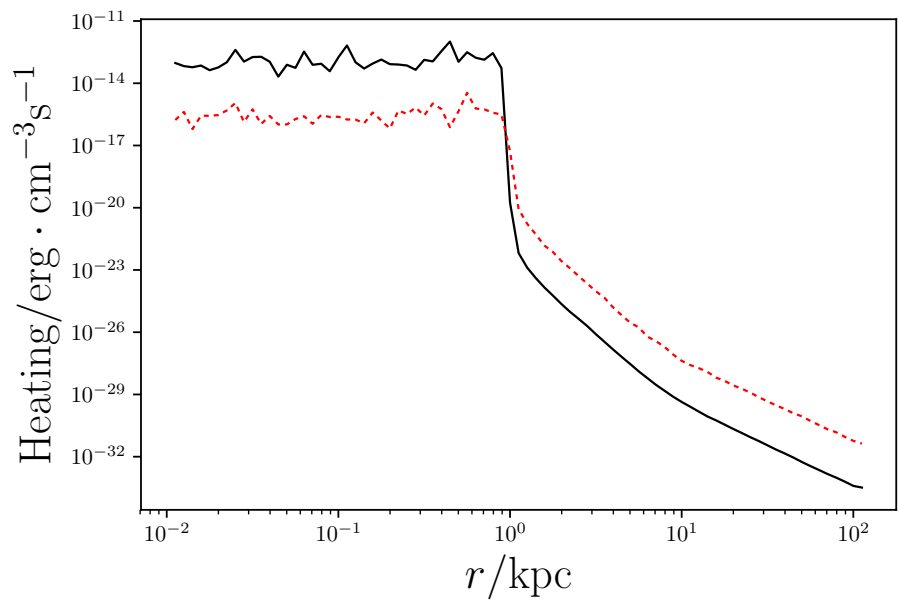

Figure 2: Heating due to CRs (black) and X-rays (red dashed) through the model the galactic magnetic field. This is the ratio between the saturated diffusion result and the freestreaming result outlined in section 2.2 as a function of position. For CR heating, the absorption parameter is defined with the cross section for the pp interaction used. For X-ray heating, the same approach may be used but here the Klein-Nishina cross section is applied instead. The results are shown in Fig. 2 for the heating profile due to the CRs (black), and X-rays (red).

\section{Summary and Conclusions}

Our calculations showed that an ISM CR heating rate may reach $10^{-13} \mathrm{erg} \mathrm{cm}^{-3} \mathrm{~s}^{-1}$ and an indirect X-ray heating effect via the SZE of $10^{-16} \mathrm{erg} \mathrm{cm}^{-3} \mathrm{~s}^{-1}$. As CR losses have not been accounted for explicitly in the transportation, these values may be considered as upper limits. Nevertheless, CR heating should not be ignored in protogalactic environments. Such heating would 
increase the Jeans mass of star-forming regions, thus distorting the initial mass function of early generations of stars. Also, the resulting X-rays may increase the ionisation fraction deep in star forming clouds, hampering the removal of magnetic fields by ambipolar diffusion [32]. Coupled with CR heating, which is expected to be particularly concentrated along the strong magnetic field vectors in star-forming regions, an amplified run-away heating effect may take hold, resulting in quenching the star formation in some regions entirely.

\section{Acknowledgements}

ERO is supported by the Science and Technology Facilities Council, a Royal Astronomical Society grant, the Institute of Physics (IoP) C R Barber Trust and the IoP Student Conference Fund.

\section{References}

[1] K. Kotera and A. V. Olinto, The Astrophysics of Ultrahigh-Energy Cosmic Rays, ARA\&A 49 (2011) 119-153, [1101.4256].

[2] I. B. Jacobsen, K. Wu, A. Y. L. On and C. J. Saxton, High-energy neutrino fluxes from AGN populations inferred from X-ray surveys, MNRAS 451 (2015) 3649.

[3] V. Berezinsky, A. Gazizov and S. Grigorieva, On astrophysical solution to ultra high energy cosmic rays, Phys. Rev. D 74 (2006) 043005.

[4] A. Dar and A. de Rújula, A theory of cosmic rays, Phys. Rep. 466 (2008) 179-241, [hep-ph/0606199].

[5] W. Dehnen, A family of potential-density pairs for spherical galaxies and bulges, MNRAS 265 (1993) $250-256$.

[6] J. Schober, D. R. G. Schleicher and R. S. Klessen, Magnetic field amplification in young galaxies, A\&A 560 (2013) A87, [1310.0853].

[7] M. Ackermann, M. Ajello, A. Allafort, L. Baldini, J. Ballet, G. Barbiellini et al., Detection of the Characteristic Pion-Decay Signature in Supernova Remnants, Science 339 (2013) 807-811.

[8] H. Benhabiles-Mezhoud, J. Kiener, V. Tatischeff and A. W. Strong, De-excitation Nuclear Gamma-Ray Line Emission from Low-energy Cosmic Rays in the Inner Galaxy, ApJ 763 (2013) 98, [1212.1622].

[9] S. Sazonov and R. Sunyaev, Preheating of the Universe by cosmic rays from primordial supernovae at the beginning of cosmic reionization, MNRAS 454 (2015) 3464-3471, [1509. 08408 ].

[10] H. Bethe and W. Heitler, On the Stopping of Fast Particles and on the Creation of Positive Electrons, Proc. R. Soc. A 146 (1934) 83-112.

[11] V. S. Berezinsky and A. Z. Gazizov, Production of high-energy cosmic neutrinos in $p \gamma$ and $n \gamma$ scattering (I), Phys. Rev. D 47 (1993) 4206-4216.

[12] S. P. Almeida, J. G. Rushbrooke, J. H. Scharenguivel, M. Behrens, V. Blobel, I. Borecka et al., pp interactions at 10 GeV/c, Phys. Rev. 174 (1968) 1638-1661. 
[13] T. Skorodko, M. Bashkanov, D. Bogoslawsky, H. Calen, F. Cappellaro, H. Clement et al., Excitation of the Roper resonance in single- and double-pion production in nucleon-nucleon collisions, Eur. Phys. J. A 35 (2008) 317-319.

[14] S. Stepney and P. W. Guilbert, Numerical FITS to important rates in high temperature astrophysical plasmas, MNRAS 204 (1983) 1269-1277.

[15] R. Jost, J. M. Luttinger and M. Slotnick, Distribution of Recoil Nucleus in Pair Production by Photons, Phys. Rev. 80 (1950) 189-196.

[16] H. A. Bethe and L. C. Maximon, Theory of Bremsstrahlung and Pair Production. I. Differential Cross Section, Phys. Rev. 93 (1954) 768-784.

[17] G. R. Blumenthal, Energy Loss of High-Energy Cosmic Rays in Pair-Producing Collisions with Ambient Photons, Phys. Rev. D 1 (1970) 1596-1602.

[18] C. D. Dermer and G. Menon, High energy radiation from black holes: gamma rays, cosmic rays, and neutrinos. Princeton series in astrophysics. Princeton Univ. Press, Princeton, NJ, 2009.

[19] A. M. Atoyan and C. D. Dermer, Neutral Beams from Blazar Jets, ApJ 586 (2003) 79.

[20] E. Kafexhiu, F. Aharonian, A. M. Taylor and G. S. Vila, Parametrization of gamma-ray production cross sections for p p interactions in a broad proton energy range from the kinematic threshold to PeV energies, Phys. Rev. D 90 (2014) 123014, [1406. 7369].

[21] R. J. Protheroe and P. A. Johnson, Propagation of ultra high energy protons and gamma rays over cosmological distances and implications for topological defect models, Astropart. Phys. 4 (1996) 253-269, [astro-ph/9506119].

[22] C. Badenes, D. Maoz and B. T. Draine, On the size distribution of supernova remnants in the Magellanic Clouds, MNRAS 407 (2010) 1301.

[23] J. Schober, D. R. G. Schleicher and R. S. Klessen, X-ray emission from star-forming galaxies signatures of cosmic rays and magnetic fields, MNRAS 446 (2015) 2.

[24] D. R. G. Schleicher and R. Beck, A new interpretation of the far-infrared-radio correlation and the expected breakdown at high redshift, A\&A 556 (2013) A142.

[25] Planck Collaboration, Planck 2015 results - XIII. Cosmological parameters, A\&A 594 (2016) A13.

[26] R. A. Sunyaev and Y. B. Zeldovich, Formation of Clusters of Galaxies; Protocluster Fragmentation and Intergalactic Gas Heating, A\&A 20 (1972) 189.

[27] G. R. Blumenthal and R. J. Gould, Bremsstrahlung, Synchrotron Radiation, and Compton Scattering of High-Energy Electrons Traversing Dilute Gases, Rev. Mod. Phys. 42 (1970) 237-270.

[28] G. B. Rybicki and A. P. Lightman, Radiative processes in astrophysics. Wiley, 1979.

[29] B. C. Lacki and R. Beck, The equipartition magnetic field formula in starburst galaxies: accounting for pionic secondaries and strong energy losses, MNRAS 430 (2013) 3171.

[30] B. C. Lacki, T. A. Thompson, E. Quataert, A. Loeb and E. Waxman, On the GeV and TeV Detections of the Starburst Galaxies M82 and NGC 253, ApJ 734 (2011) 107, [1 003 . 32 57].

[31] S. X. Wang, W. N. Brandt, B. Luo, I. Smail, D. M. Alexander, A. L. R. Danielson et al., An ALMA Survey of Submillimeter Galaxies in the Extended Chandra Deep Field-South: The AGN Fraction and X-Ray Properties of Submillimeter Galaxies, The Astrophysical Journal 778 (2013) 179.

[32] B. T. Draine, Physics of the Interstellar and Intergalactic Medium. Princeton University Press, 2011. 